\title{
UTILIZAÇÃO DE PREVISÕES DE PRECIPITAÇÃO DE MODELOS ATMOSFÉRICOS WRF, GFS E GEFS NA BACIA HIDROGRÁFICA DO RIO AVE (PORTUGAL) PARA GESTÃO OPERACIONAL DE UM SISTEMA DE DRENAGEM
}

\author{
USE OF PRECIPITATION FORECASTS FROM WRF, GFS AND GEFS ATMOSPHERIC \\ MODELS AT RIVER AVE BASIN (PORTUGAL) FOR OPERATIONAL MANAGEMENT OF A \\ DRAINAGE SYSTEM
}

\author{
José Luis da Silva Pinho ${ }^{a}$, António Pereira ${ }^{a b}$, Rolando Faria ${ }^{b}$

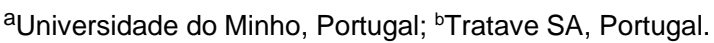 \\ e-mail: ppinho@civil.uminho.pt, antonio.pereira@tratave.pt, rolando.faria@tratave.pt
}

Submissão: 30 de abril de 2020 Aceitação: 20 de setembro de 2020

\section{Resumo}

Os sistemas de previsão e alerta utilizados na gestão de recursos hídricos e operação de sistemas de drenagem tiveram desenvolvimentos significativos nos últimos anos. Esses desenvolvimentos resultaram da disponibilidade de informações meteorológicas em tempo real, em particular de medições por sensores em satélites, medição através de radar meteorológico e de previsões de modelos atmosféricos para diferentes horizontes temporais. Todos os modelos de previsão ambiental são incertos e essa incerteza é variável no tempo e no espaço. Este trabalho tem como objetivo apresentar os resultados da avaliação da evolução do erro associado a diferentes previsões de curto prazo. A plataforma Delft-FEWS (Flood Early Warning System) foi utilizada para proceder à importação e processamento de dados de observações e previsões disponíveis para a bacia do rio Ave, localizada no norte de Portugal. Os dados meteorológicos medidos foram obtidos no Sistema Nacional de Informações de Recursos Hídricos (SNIRH), em quatro estações meteorológicas instaladas na bacia em estudo e dados de refletividade medidos pelo radar meteorológico operado pela Meteogalicia. As previsões avaliadas correspondem às precipitações simuladas por modelos atmosféricos desenvolvidos pela National Oceanic and Atmospheric Administration (NOAA) e Meteogalicia, nomeadamente os modelos Global Forecast System (GFS), Global Ensemble Forecast System (GEFS) e Weather Research and Forecasting, (WRF) operado pela Meteogalicia. A incerteza associada às precipitações previstas foi avaliada considerando horizontes de previsão de um a quatro dias. Os melhores resultados foram obtidos para o modelo WRF durante eventos de precipitação ocorridos entre janeiro de 2017 e maio de 2018 e apresentaram médias de erros relativos que variaram entre $7 \%$ (um dia de previsão) e $29 \%$ (quatro dias). 0 sistema implementado permite, assim, do ponto de vista operacional, antecipar com antecedência de dois dias eventos extremos.

Palavras-chave: Previsões de precipitação; sistemas de alerta; análise da incerteza; hidroinformática; bacia do rio Ave.

\section{Abstract}

The forecasting and warning systems used in water resources management and drainage systems operation have had significant developments in recent years. These developments resulted from the availability of meteorological information in real time, in particular from measurements by sensors in satellites, measurement through meteorological radar and forecasts of atmospheric models for different time horizons. All environmental forecasting models are uncertain and this uncertainty varies over time and space. This work aims to present the results of the evaluation of the evolution of the error associated with different short-term forecasts. The Delft-FEWS (Flood Early Warning System) platform was used to import and process observation and forecast data available for the river Ave basin, located in northern Portugal. The measured meteorological data were obtained from the National Water Resources Information System (SNIRH), at four new meteorological stations installed in the basin and radar reflectivity data measured by the meteorological 
radar operated by Meteogalicia. The forecasts evaluated correspond to the rainfall simulated by atmospheric models developed by the National Oceanic and Atmospheric Administration (NOAA) and Meteogalicia, namely the Global Forecast System (GFS), Global Ensemble Forecast System (GEFS) and Weather Research and Forecasting (WRF) model operated by Meteogalicia. The uncertainty associated with the predicted rainfall was evaluated considering forecast horizons of one to four days. The best results were obtained for the WRF model during precipitation events that occurred between January 2017 and May 2018 and presented average relative errors that varied between $7 \%$ (one forecast day) and $29 \%$ (four days). The implemented system thus allows, from an operational point of view, to forecast extreme events in advance of two days.

Keywords: Precipitation forecasts; warning systems; uncertainty analysis; hydroinformatics; river Ave basin.

\section{INTRODUÇão}

A operação de sistemas de drenagem durante a ocorrência de eventos extremos obriga à mobilização de meios técnicos e humanos que permitam uma resposta eficaz, sempre que surjam obstruções ou se ultrapasse a capacidade de drenagem instalada. Os impactos negativos provenientes destes dois tipos de ocorrências tornam o estudo e implementação de ferramentas que permitam a sua antecipação cada vez mais importante. A modelação matemática é uma ferramenta essencial, constituindo uma metodologia eficiente e útil para a análise, estudo e previsão de cheias e inundações em sistemas naturais ou derrames em infraestruturas de drenagem. Contudo, quando utilizadas em modo de previsão, a qualidade dos resultados depende da incerteza associada aos dados de precipitação utilizados para forçar os modelos.

As condições meteorológicas, nomeadamente as previsões de curto e médio termo de modelos atmosféricos, são de extrema importância para os processos de suporte à decisão (Butler e Schutze, 2005; Fletcher et al., 2013; Joseph-Duran et al., 2014). A utilização de ferramentas avançadas hidroinformáticas é ainda pouco explorada na prática corrente da operação de sistemas de drenagem. De referir que em termos académicos, tem-se assistido a avanços extremamente representativos, não obstante, as entidades gestoras mostram-se ainda pouco recetivas à utilização deste tipo de ferramentas na gestão operacional das suas infraestruturas.

Pasqual et al. (2016) referem que a previsão meteorológica se tornou fundamental não apenas na relação do homem com o meio ambiente, mas também no desenvolvimento de diversas atividades económicas. Atualmente, são utilizados modelos de previsão numérica baseados numa vasta quantidade de dados e no uso de tecnologias avançadas para monitorizar a atmosfera terrestre.

Existe um interesse crescente (Pinho e Vieira,
2014; Joseph-Duran et al., 2014 e Lowe et al., 2016) em desenvolver e aplicar metodologias de gestão operacional de recursos hídricos, especialmente utilizando ferramentas de controlo em tempo real (RTC) (Fuchs e Beeneken, 2005; Puig, 2009; Pinho et al., 2011; Lowe et al., 2016; Van Daal-Rombouts et al., 2016; Shishegar et al., 2018; Pereira et al., 2018; Pereira et al., 2019).

A disponibilização de resultados de previsões meteorológicas e monitorização de variáveis hidrológicas obrigam à disponibilização de ferramentas que permitam a sua importação e processamento de modo eficiente. O desenvolvimento de um sistema de previsão e alerta eficaz depende do sucesso na integração de grandes conjuntos de dados, módulos especializados para o respetivo processamento e interfaces que permitam uma fácil integração das capacidades de modelação existente. Em resposta a estes desafios, a plataforma Delft-FEWS (Deltares, 2011) foi desenvolvida para implementar sistemas de previsão hidrológica e sistemas de alerta. Esta plataforma é composta por um conjunto de módulos que permitem a construção de sistemas de previsão hidrológica adaptados às necessidades específicas de cada um.

Este trabalho tem como objetivo apresentar os resultados da avaliação da evolução do erro associado a diferentes previsões de precipitações de curto prazo. De facto, a incerteza associada às previsões disponíveis atualmente permitem criar plataformas operacionais eficientes para horizontes de previsão da ordem de vários dias (Schwanenberg et al., 2013, 2015). Contudo, a incerteza associada às previsões é variável espacialmente (ex., Medina et al., 2019). A plataforma Delft-FEWS (Flood Early Warning System) foi utilizada para proceder à importação e processamento de dados de observações e previsões disponíveis para a bacia do rio Ave, localizada no norte de Portugal. O trabalho foi desenvolvido em parceria com a empresa 
responsável pela gestão operacional das infraestruturas de drenagem e tratamento de águas residuais da bacia do rio Ave (TRATAVE) e seus afluentes que opera e gere uma rede de intercetores que se desenvolve ao longo de 320 $\mathrm{km}$ em cinco municípios: Guimarães, Vizela, Famalicão, Trofa e Santo Tirso.

Os dados meteorológicos medidos foram obtidos no Sistema Nacional de Informações de Recursos Hídricos (SNIRH), em quatro estações meteorológicas instaladas na bacia em estudo e dados de refletividade medidos pelo radar meteorológico operado pela Meteogalicia. As previsões avaliadas correspondem às precipitações simuladas por modelos atmosféricos desenvolvidos pela National Oceanic and Atmospheric Administration (NOAA) e Meteogalicia, nomeadamente os modelos Global Forecast System (GFS), Global Ensemble Forecast System (GEFS) e Weather Research and Forecasting, (WRF) operado pela Meteogalicia. A incerteza associada às precipitações previstas foi avaliada considerando horizontes de previsão de um a quatro dias.

\section{METODOLOGIA}

\subsection{Caracterização da Área em Estudo}

O âmbito geográfico deste trabalho é a bacia hidrográfica do rio Ave (Figura 1), que inclui uma densa rede de linhas de água delimitadas a norte pela bacia do rio Cávado, a este pela bacia do rio Douro e a sul pelas bacias dos rios Leça e Douro. O Rio Ave nasce na serra da Cabreira, concelho de Vieira do Minho, a 1200 m de altitude, percorrendo $94 \mathrm{~km}$ no sentido nascente-poente até à foz no Oceano Atlântico junto à cidade de Vila do Conde. A bacia possui uma área total de $1469 \mathrm{~km}^{2}$, sendo que $247 \mathrm{~km}^{2}$ e $380 \mathrm{~km}^{2}$ correspondem às áreas das bacias de seus principais afluentes, os rios Este e Vizela, respetivamente. Os principais intercetores acompanham a rede fluvial transportando os efluentes até estações de tratamento de águas residuais (ETAR). Em relação ao clima, a região é caracterizada por verões muito quentes e levemente húmidos e invernos frios e chuvosos, apresentando precipitações médias anuais superiores a $1500 \mathrm{~mm}$. A intensa precipitação que ocorre nos períodos chuvosos, juntamente com as características orográficas, a constituição do solo das bacias e sua permeabilidade, tornam esta zona propícia a inundações e cheias.
Esta massa de água engloba, total ou parcialmente, as áreas de jurisdição de 16 concelhos. Os concelhos de Guimarães e Vila Nova de Famalicão ficam totalmente integrados na bacia hidrográfica do rio Ave, enquanto Braga, Barcelos, Vizela, Celorico de Basto, Fafe, Póvoa do Lanhoso e Vieira do Minho, todos do distrito de Braga e ainda parte dos concelhos de Felgueiras, Lousada, Maia, Paços de Ferreira, Póvoa do Varzim, Santo Tirso e Vila do Conde do distrito do Porto.

Trata-se de uma região caraterizada por boas capacidades de recarga natural provenientes dos cursos de água ou por infiltração da água da chuva. No caso dos aquíferos formados por estratos distintos a recarga acontece a partir da drenagem de camadas adjacentes. A paisagem ribeirinha do Rio Ave é ainda extremamente valorizada pelo grande número de pontes romanas e medievais que o atravessa, pela existência de elementos com interesse arquitetónico e paisagístico, bem como pelos inúmeros exemplares de azenhas e açudes.

A bacia hidrográfica é monitorizada por uma rede meteorológica que serve para acompanhar o ramo aéreo do ciclo hidrológico (precipitação, temperatura, humidade do ar, direção e velocidade do vento, insolação, radiação solar, evaporação e evapotranspiração), sendo constituída por 59 estações, das quais cinco são climatológicas e as restantes são pluviométricas (neste trabalho utilizam-se 35 destas estações). Existe ainda uma rede hidrométrica utilizada para monitorizar o nível hidrométrico, a partir do qual são recolhidos dados de escoamento (caudal/vazão, nível, velocidade), sendo possível acompanhar de forma mais eficaz o comportamento dos rios. São monitorizados os níveis freáticos, para posterior classificação do estado quantitativo e da condutividade e concentrações de poluentes para determinação do estado químico.

Embora a rede de monitorização seja extensa muitas das estações encontram-se desativadas e outras em fase de manutenção, incluindo apenas dados históricos. Por outro lado, o desenvolvimento de um sistema de suporte à decisão necessita de dados disponíveis em tempo real com frequências de registo iguais ou inferiores à horária.

Assim, instalaram-se quatro estações meteorológicas em locais distintos da bacia, três equipamentos para monitorização de caudal em diferentes trechos dos rios Ave e Vizela e diversos equipamentos de medição da altura de 
escoamento nos intercetores de drenagem de águas residuais (Figura 2). Estes sensores permitem acompanhar a evolução dos caudais recolhidos, incluindo os que resultam de ligações indevidas de águas pluviais à rede de drenagem de águas residuais. Foram ainda configurados procedimentos que permitem processar os dados medidos pela rede de monitorização instalada, sendo a informação disponibilizada em tempo real.

Figura 1: Localização da bacia do rio Ave.

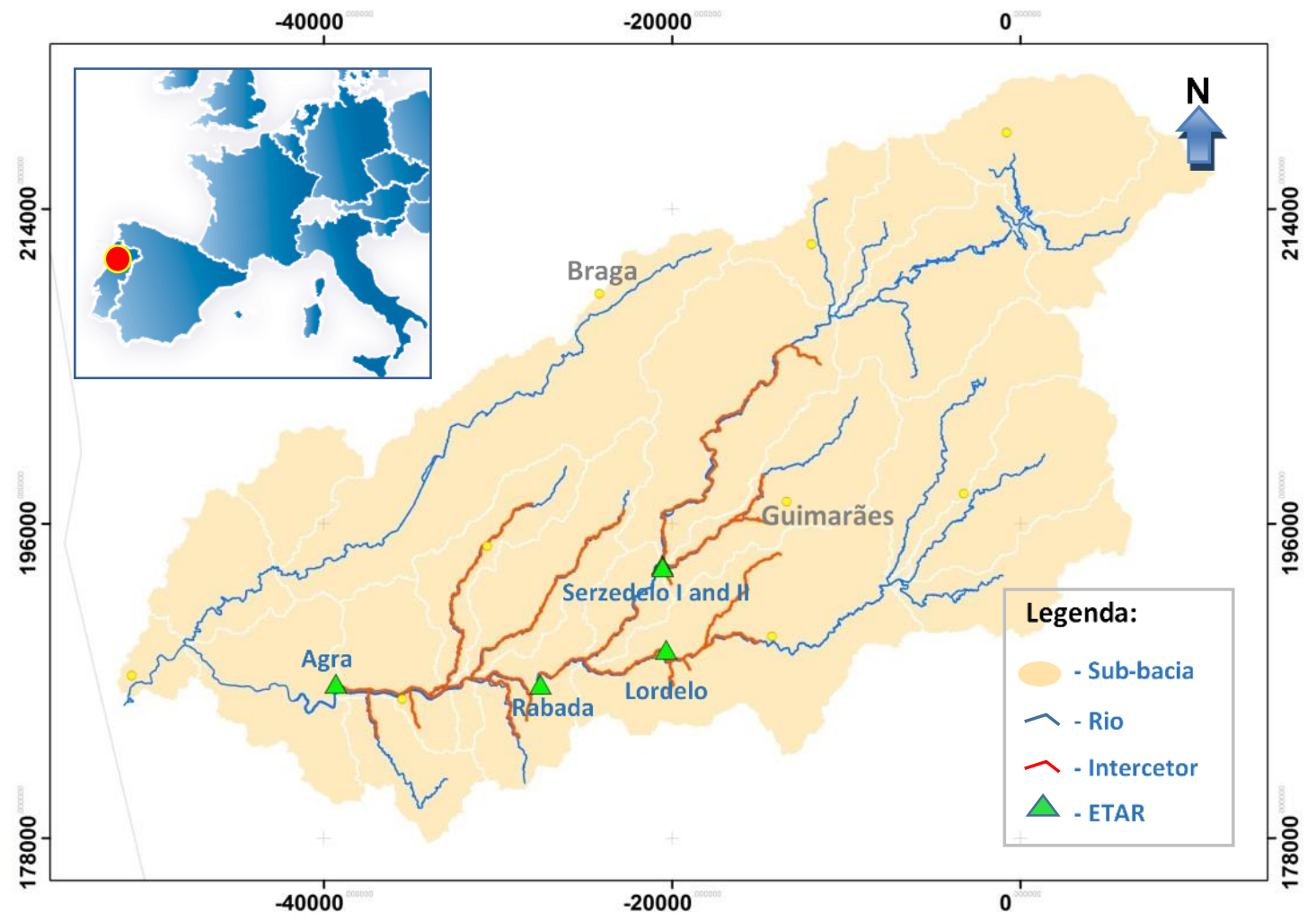

Fonte: Autores, 2020.

\subsection{Sistema de apoio à decisão}

Os dados utilizados neste trabalho foram processados no âmbito do desenvolvimento de um sistema de apoio à decisão (SAD) para a gestão operacional do sistema de drenagem e tratamento da TRATAVE. Foi utilizada a plataforma DelftFEWS que para além dos dados de base (componente de informação) está a ser utilizada para implementar uma componente de modelação que permita gerar previsões com base em estados atualizados dos sistemas simulados recorrendo-se a técnicas de assimilação de dados. Foram inseridos os dados históricos disponíveis no SNIRH e os dados de quatro estações meteorológicas operadas pela TRATAVE. Foram ainda utilizados os dados de Radar, fornecidos pelo servidor THREDDS (Thematic Realtime Environmental Distributed Data Service) da MeteoGalicia.
O SAD (Figura 3), tem como suporte o sistema operativo Windows Server 2012R2 e está instalado num datacenter, sob a gestão da Altice (cloud server). A informação dos sensores é gerida e disponibilizada por um sistema SCADA (Supervisory Control And Data Acquisition). Os dados são armazenados numa base de dados criada com o software Microsoft SQL Server, sendo partilhados através de protocolos FTP (File Transfer Protocol). O sistema apresenta três componentes principais: (i) componente de informação que inclui todos os dados de monitorização, características das infraestruturas e os dados relevantes de gestão operacional; (ii) modelo hidrológico, hidrodinâmico e de qualidade de água, integrando linhas de água e rede de drenagem de águas residuais; (iii) componente responsável pela análise e pelo processamento dos dados que apoiam a operação do sistema. 
Figura 2: Equipamentos de monitorização.
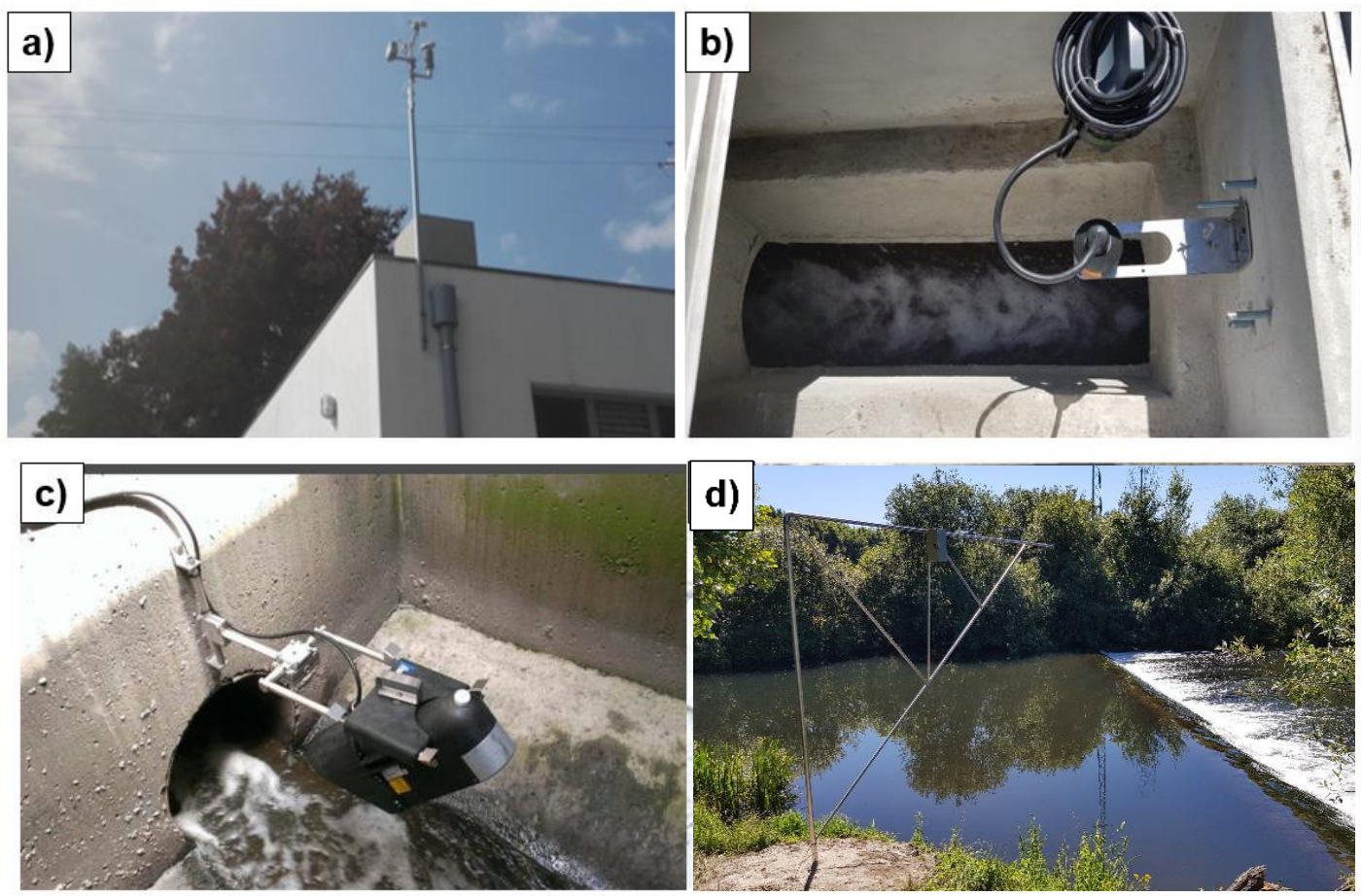

a) estação meteorológica PCE, b) sensor ultrassónico Sofrel LT US, c) equipamento doppler Laserflow e d) estrutura com instalação de sensor Sofrel LT US em estação fluvial.

Fonte: Autores, 2020

Os modelos são operados recorrendo-se à plataforma Delft-FEWS. A sua calibração foi baseada em dados meteorológicos, hidrológicos e de caudais das linhas de água obtidos através do SNIRH e da rede de monitorização da TRATAVE. As capacidades de previsão foram implementadas de forma a antecipar diferentes eventos de precipitação, melhorando-se, assim, o desempenho da empresa na mitigação de problemas resultantes de volumes infiltrados que são transportados para as ETAR, sobretudo durante ocorrência de eventos de precipitação intensa.

A previsão de eventos de precipitação é caracterizada por um elevado grau de incerteza. Para atenuar e avaliar essa incerteza, embora de modo qualitativo, recorre-se aos dados de precipitação de três modelos atmosféricos distintos. Geralmente é considerada uma boa prática considerar um maior número de modelos de modo a permitir a comparação entre os diferentes resultados obtidos. Em qualquer caso, os dados devem estar atualizados para que a previsão seja tão precisa quanto possível.
Para previsões de precipitações recorreu-se ao modelo atmosférico WRF que tal como os dados de Radar, são processados pelo servidor THREDDS da MeteoGalicia, entidade que também é responsável por este modelo operacional. Recorre-se ainda aos resultados do modelo GFS disponibilizados pela NOAA e aos resultados GEFS, disponibilizados pela mesma entidade.

$\mathrm{Na}$ figura 4 apresentam-se as principais fontes de informação utilizadas no trabalho, nomeadamente a localização de rede de monitorização do SNIRH (Figura 4-a) e dados do radar meteorológico da Meteogalicia (Figura 4-b).

O modelo GFS da NOAA apresenta previsões para um horizonte de 10 dias com uma resolução espacial de $0.1^{\circ}$ Ion $\times 0.1^{\circ}$ lat e uma resolução temporal de $6 \mathrm{~h}$. O modelo WRF, apresenta uma previsão para 4 dias com uma resolução espacial de $4 \mathrm{~km} \times 4 \mathrm{~km}$ e resolução temporal horária para uma região que cobre a Galiza e o norte de Portugal e por fim o terceiro modelo, GEFS, apresenta 21 membros com previsão para 16 dias, uma resolução espacial de $27 \mathrm{~km} \times 27 \mathrm{~km}$ e resolução temporal de $6 \mathrm{~h}$. 
Figura 3: Principais componentes do SAD.

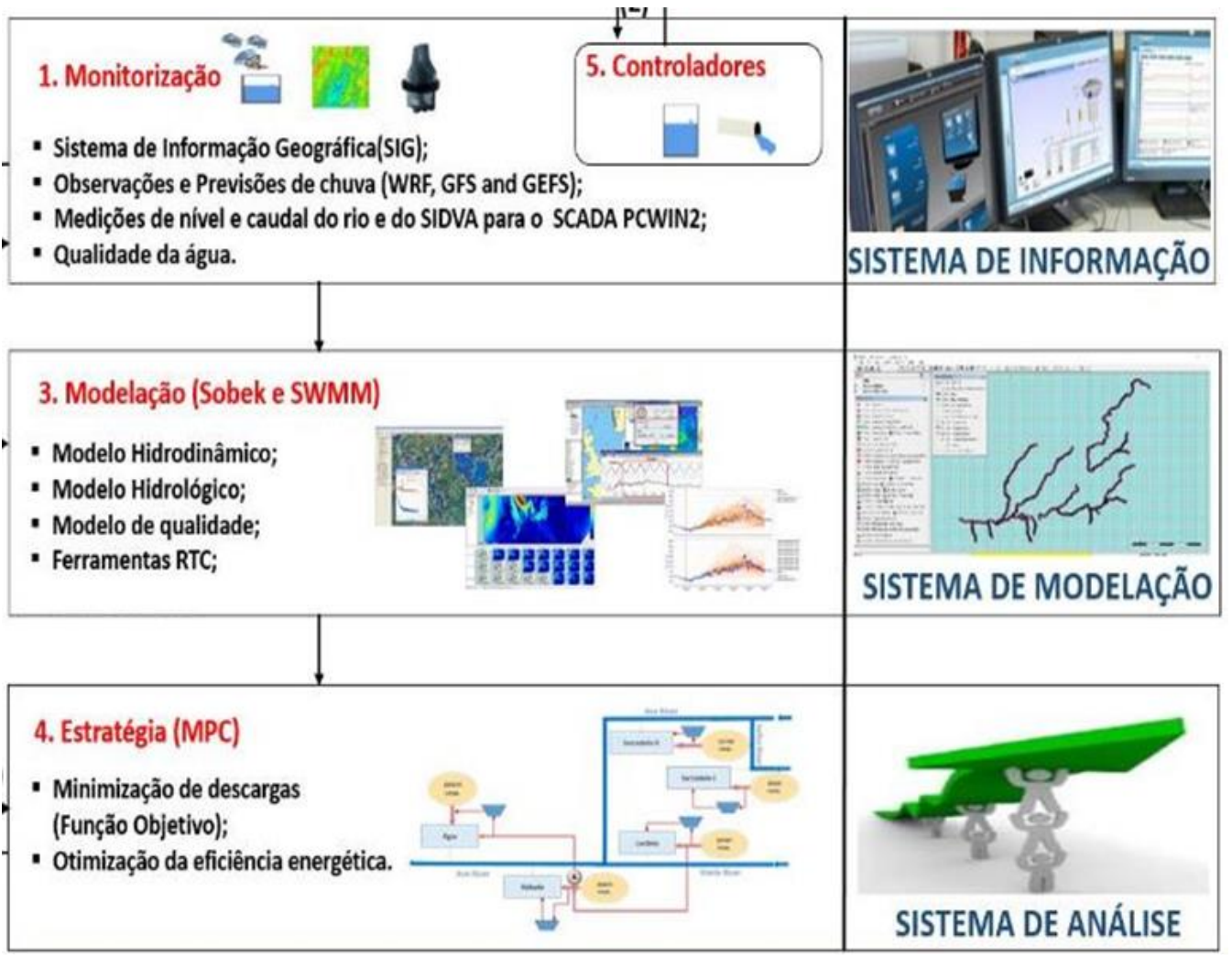

Fonte: Autores, 2020.

Figura 4: Fontes de informação para a construção do SAD (Delft-FEWS).
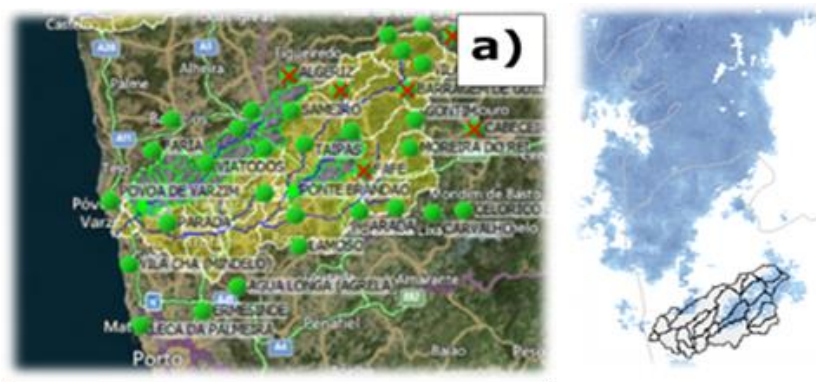

b)

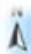

c)

$\ddot{i}$

d)

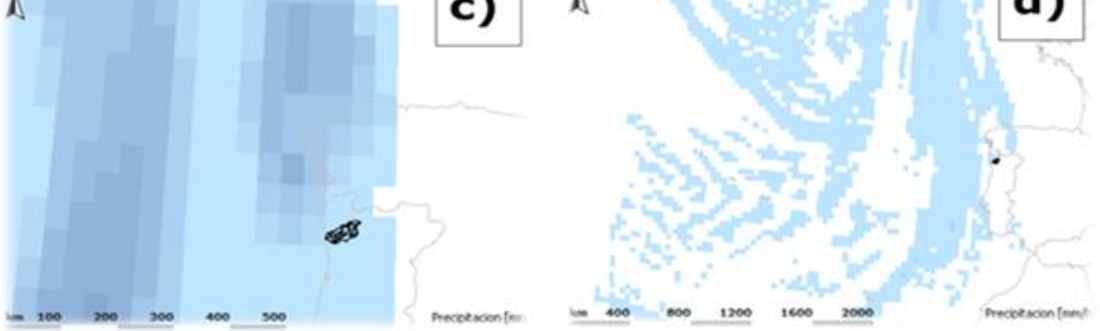

a) Rede de monitorização do SNIRH, b) Radar da Meteogalicia, c) Um dos modelos de previsão da NOOA - GFS, d) Modelo de previsão da Metogalicia - WRF.

Fonte: Autores, 2020. 


\subsection{Processamento das medições de radar}

O radar meteorológico mede a refletividade (dBz) que se relaciona com a precipitação, sendo necessário converter o fator de refletividade $(Z)$, em intensidade de precipitação $(R)$. É importante salientar que Z e R são quantidades diferentes, embora estejam relacionadas com o mesmo fenómeno físico. O fator de refletividade $(Z)$ depende da quantidade e dimensão das gotas de água presentes no volume iluminado pelo radar e o $R$ representa a precipitação que atinge uma área de um determinado local. Marshall e Palmer (1984) propuseram a primeira equação baseada em muitas observações experimentais, relacionando as informações obtidas por radar e medidas em udómetros. Vários trabalhos têm vindo a ajustar as equações $Z=f(R)$ para diferentes regiões e tipos de precipitação (Kaiser, 2006). A relação clássica entre precipitação de radar e refletividade é apresentada na eq. (1):

$$
Z=A R^{B}
$$

onde,

A e B: parâmetros ajustáveis;

$Z$ é o fator de refletividade $(\mathrm{dBz})$;
R é a intensidade de precipitação ( $\mathrm{mm} / \mathrm{h})$.

No Quadro 1 são apresentados os valores dos parâmetros usualmente utilizados na equação de transformação dos valores de refletividade resultantes de medições de radar em precipitações para diferentes tipologias de precipitação.

Neste estudo, os valores dos parâmetros A e $B$ foram estimados a partir de 2867 registos de precipitação com valores entre $0,3 \mathrm{~mm}$ e $7 \mathrm{~mm}$ e resolução temporal de 5 minutos, medidos durante o período de janeiro de 2017 a maio de 2018 .

O ajuste conseguido é avaliado para duas estações adicionais distintas da utilizada para obter os valores dos parâmetros da lei de transformação da refletividade em precipitação.

Os dados de radar, após o ajuste realizado, são processados automaticamente na plataforma operacional desenvolvida, tendo sido configurado o procedimento com base nos valores dos parâmetros obtidos. Deste modo, é possível disponibilizar dados de precipitação para a bacia com elevada resolução espacial e temporal, permitindo avaliar a qualidade das previsões à posteriori e relacionar os dados de exploração do sistema com os eventos de precipitação medidos pelo radar meteorológico.

Quadro 1 - Parâmetros da equação de Marshal e Palmer (1948) para diferentes tipos de precipitação (adaptado de Kaiser, 2006)

\begin{tabular}{l|c|c|l}
\hline \multicolumn{1}{c|}{ Referência } & & & \\
\hline Marshall \& Palmer (1948) & A & B & \multicolumn{1}{|c}{ Tipo de precipitação } \\
Blanchard (1953) & 200 & 1.60 & Precipitações homogéneas e estratiformes \\
Jones (1956) & 31 & 1.71 & Precipitação orográfica \\
Joss \& Waldvogel (1967) & 483 & 1.37 & Precipitação convetiva \\
Joss \& Waldvogel (1967) & 230 & 1.40 & Precipitações médias \\
Joss \& Waldvogel (1967) & 400 & 1.30 & Processos convetivos intensos \\
Smith et al. (1975) & 100 & 1.40 & Processos frontais - gotas muito pequenas \\
\hline
\end{tabular}

\section{RESULTADOS E DISCUSSÃO}

\subsection{Transformação de dados de radar em precipitação}

Foi realizado o ajuste dos valores dos parâmetros da equação de Marshal e Palmer (1948), a partir dos valores de precipitação registados na estação meteorológica da ETAR de
Serzedelo e dos valores de refletividade do radar operado pela Meteogalicia, interpolados para o mesmo local.

Os resultados apresentados na figura 5 mostram a relação entre os dados medidos pela estação meteorológica e os estimados a partir das medições de refletividade do radar com valores dos parâmetros de $A=263$ e $B=1.43$. 
$O$ ajuste conseguido (coeficiente de determinação $R^{2}=0.93$ ) é aceitável atendendo a que foram estimados com base numa série de dados horários. Os valores dos parâmetros obtidos estão dentro do intervalo de variação que resulta de outros trabalhos (Quadro 1). O valor do parâmetro A é próximo do valor proposto por Joss \& Waldvogel (1967) para precipitações médias e o valor do parâmetro B próximo dos valores propostos pelos mesmos autores para precipitações médias e processos frontais - gotas muito pequenas. Os valores ajustados não são muito distintos dos valores propostos por Marshall \& Palmer (1948) para precipitações homogéneas e estratiformes. As tipologias de precipitações na bacia do rio Ave serão próximas das indicadas, apresentando uma forte influência Atlântica (Nicolau, 2002).

Contudo, nem sempre se poderá assumir como válido que os parâmetros da equação de transformação de refletividade para intensidade de precipitação se mantêm constantes para todo o intervalo de variação da intensidade de precipitação, sobretudo para os eventos de precipitação mais intensos (ex., Wu et al., 2018). Para se avaliar o desempenho da equação de transformação adotada foram obtidos dados de precipitação para duas estações adicionais
(Estação elevatória de Lagoas e ETAR de Agra). $O$ desempenho da equação de transformação foi avaliado para os registos de radar e precipitações horárias do ano de 2019. Neste ano registaram-se vários eventos de precipitação intensa $(>5 \mathrm{~mm} / \mathrm{h})$ com interesse para a gestão operacional das infraestruturas de drenagem uma vez que implicam funcionamento em pressão, com transbordamentos em trechos significativos da rede.

Os resultados para o ano de 2019 são apresentados na figura 6 . As estações localizamse a este (EE Lagoas) e a oeste (ETAR de Agra) da estação de Serzedelo utilizada para efetuar o ajuste da lei de transformação. Os resultados obtidos indiciam dois aspetos importantes na utilização futura da equação de transformação: i) o ajuste é adequado para utilização em toda a extensão das infraestruturas de drenagem conforme se comprova pelos valores dos coeficientes de determinação obtidos e ii) o ajuste é adequado para os eventos de precipitação intensos responsáveis, como referido anteriormente, pelo funcionamento em pressão da rede. Deste modo, poderão estes dados ser utilizados na análise e operação das infraestruturas, tirando-se partido da sua elevada frequência.

\section{Figura 5: Precipitação estimada a partir dos valores de refletividade do radar operado pela Meteogalicia após ajuste dos parâmetros da equação de Marshal-Palmer, tendo por base os valores registados na estação meteorológica da ETAR de Serzedelo.}

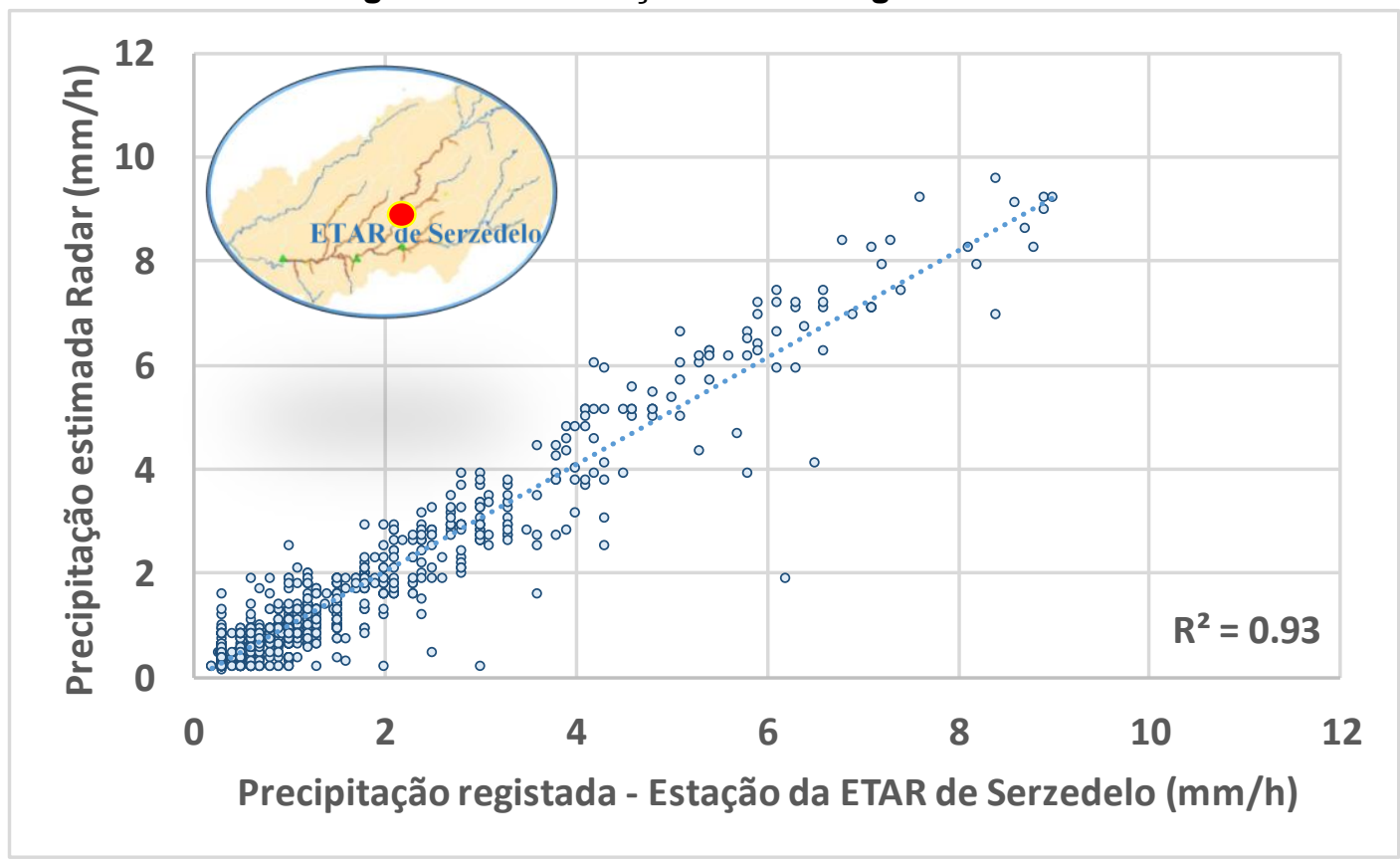

Fonte: Autores, 2020. 
Figura 6: Avaliação do desempenho da equação de transformação adotada para duas estações meteorológicas distintas: estação meteorológica da elevatória de Lagoas (superior) com um $R^{2}=0.97$ e estação meteorológica da ETAR de Agra com um $R^{2}=0.98$ (inferior).
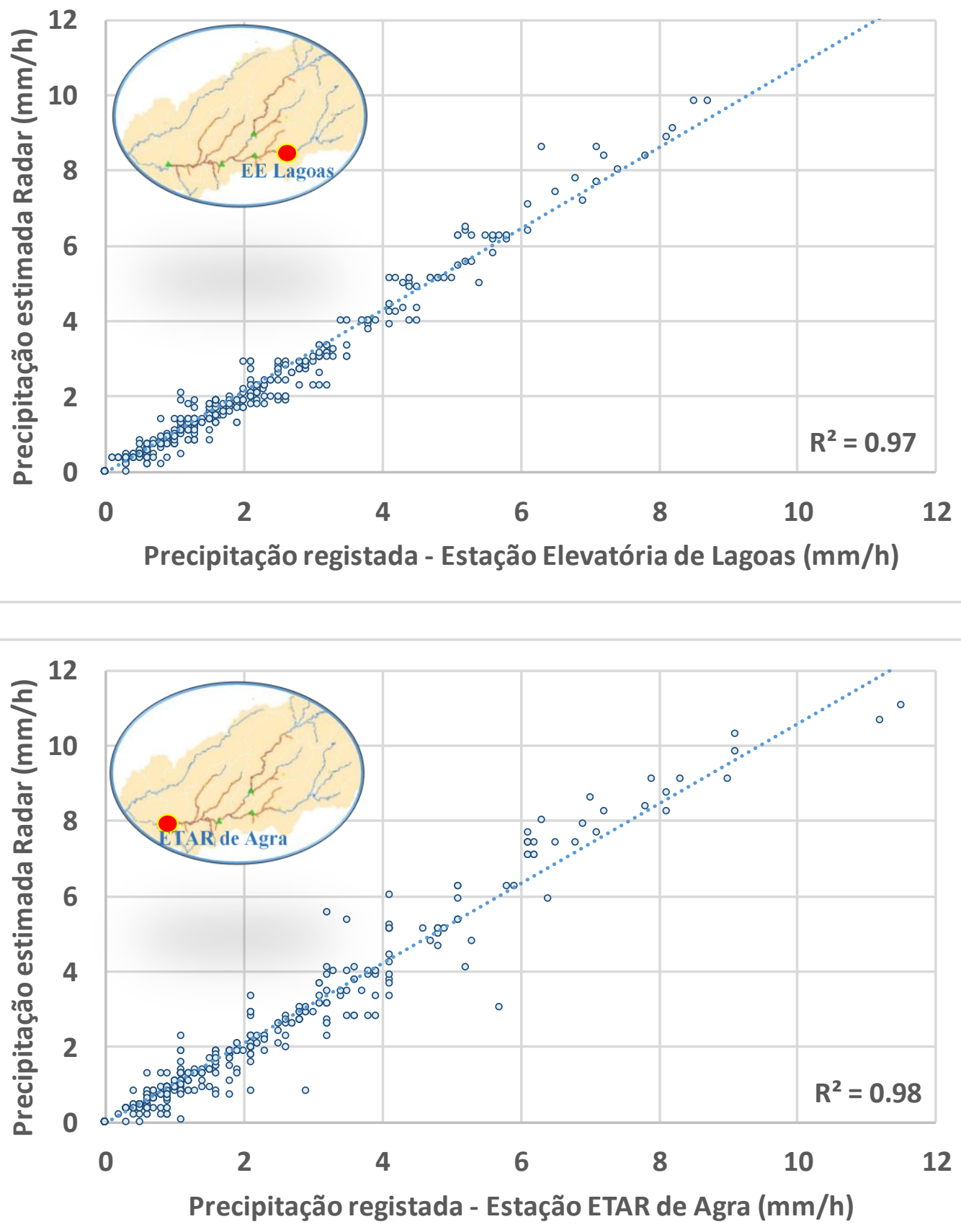

Fonte: Autores, 2020.

Pode-se ainda assumir que os valores dos registos de radar poderão ser utilizados para outras localizações intermédias da bacia, permitindo uma caracterização dos eventos de precipitação com maior resolução espacial do que a que resulta do sistema de monitorização através das estações udométricas instaladas.

\subsection{Evolução do erro das previsões dos} modelos GFS, GEFS e WRF

Para além dos registos históricos de precipitação na gestão operacional do sistema de drenagem, é absolutamente crucial dispor de previsões com diferentes horizontes para se poder 
adotar medidas de mitigação adequadas no caso de ocorrência de eventos intensos.

Para tal, foi realizada uma análise dos dados medidos e dos respetivos valores previstos por diferentes modelos de previsão (GFS, GEFS - $1^{\circ}$ ำ membro e WRF) para horizontes temporais entre um e quatro dias.
Os valores diários das precipitações registadas na estação de Serzedelo e os valores de previsão são apresentadas no Quadro 2 para 14 eventos ocorridos nos anos de 2017 e 2018. Constata-se que o modelo WRF apresenta o melhor desempenho, para todos os horizontes temporais.

\section{Quadro 2: Comparação entre registos de precipitação medidos na estação de Serzedelo e valores previstos com modelos atmosféricos para 14 eventos ocorridos nos anos de 2017 e 2018.}

\begin{tabular}{|c|c|c|c|c|c|c|c|c|c|c|c|c|c|}
\hline \multirow{3}{*}{ Data } & \multirow{3}{*}{ 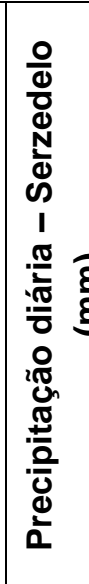 } & \multicolumn{12}{|c|}{ Horizonte de previsão } \\
\hline & & \multicolumn{3}{|c|}{1 dia } & \multicolumn{3}{|c|}{2 dias } & \multicolumn{3}{|c|}{3 dias } & \multicolumn{3}{|c|}{4 dias } \\
\hline & & WRF & GFS & GEFS & WRF & GFS & GEFS & WRF & GFS & GEFS & WRF & GFS & GEFS \\
\hline 02-02-2017 & 21.3 & 24.0 & 26.4 & 26.4 & 24.0 & 26.6 & 26.6 & 31.6 & 38.6 & 37.0 & 33.0 & 41.4 & 40.1 \\
\hline 03-03-2017 & 18.4 & 18.0 & 19.8 & 20.2 & 19.8 & 22.2 & 22.2 & 21.0 & 26.0 & 28.0 & 23.0 & 32.0 & 30.0 \\
\hline $30-04-2017$ & 14.0 & 14.0 & 16.0 & 16.3 & 14.3 & 20.3 & 19.3 & 18.0 & 28.0 & 21.0 & 23.0 & 28.0 & 31.0 \\
\hline $11-05-2017$ & 33.0 & 32.0 & 39.0 & 35.0 & 37.0 & 43.0 & 41.0 & 26.0 & $\mid 19.0$ & 27.0 & 41.0 & 45.0 & 43.0 \\
\hline $28-05-2017$ & 13.0 & 14.6 & 16.2 & 15.2 & 16.2 & 16.4 & 15.8 & 18.0 & 28.0 & 24.0 & 21.0 & 24.0 & 26.0 \\
\hline $18-10-2017$ & 27.2 & 26.0 & 24.0 & 24.0 & 26.0 & 23.4 & 21.1 & 29.3 & 29.6 & 29.0 & 30.2 & 34.0 & 33.6 \\
\hline $31-12-2017$ & 25.0 & 24.0 & 25.0 & 23.0 & 25.3 & 28.0 & 26.0 & 27.0 & 30.0 & 29.0 & 31.0 & 32.0 & 32.0 \\
\hline $10-01-2018$ & 22.0 & 24.1 & 17.1 & 19.0 & 26.8 & 14.2 & 15.0 & 28.6 & 14.8 & 15.7 & 29.0 & 12.1 & 13.8 \\
\hline $27-02-2018$ & 39.6 & 35.2 & 43.4 & 46.0 & 35.0 & 42.0 & 43.0 & 37.0 & 43.0 & 43.0 & 36.3 & 41.4 & 44.0 \\
\hline 04-03-2018 & 14.1 & 13.6 & 15.9 & 15.1 & 13.1 & 15.2 & 15.0 & 16.8 & 20.3 & 19.0 & 18.9 & 23.4 & 26.0 \\
\hline $19-03-2018$ & 16.9 & 18.1 & 21.0 & 22.0 & 18.9 & 21.6 & 22.0 & 20.0 & 23.0 & 23.0 & 21.0 & 24.0 & 21.0 \\
\hline 03-04-2018 & 57.3 & 62.6 & 45.0 & 47.0 & 64.6 & 41.0 & 38.0 & 59.8 & 70.0 & 73.0 & 64.0 & 79.0 & 76.0 \\
\hline 04-04-2018 & 22.3 & 19.8 & 26.0 & 24.0 & 21.0 & 21.0 & 24.0 & 22.0 & 22.0 & 26.0 & 24.0 & 24.0 & 23.0 \\
\hline $25-05-2018$ & 25.1 & 27.6 & 24.0 & 23.0 & 28.0 & 25.0 & 24.0 & 28.6 & 34.0 & 33.0 & 30.6 & 36.0 & 33.0 \\
\hline
\end{tabular}

$\mathrm{Na}$ figura 7 apresentam-se as médias dos erros relativos para cada um dos modelos analisados e para os quatro horizontes temporais considerados. Pode-se verificar que o modelo WRF apresenta o melhor desempenho e que o erro, como seria expectável, vai aumentando até ao quarto dia de previsão. No entanto, os resultados revelam um desempenho adequado à sua utilização na gestão operacional, com uma média do erro relativo para as previsões do modelo WRF inferior a $11 \%$ para dois de previsão, inferior a $20 \%$ para três dias e a $30 \%$ para quatro dias.
O modelo WRF possui o melhor desempenho quando comparado com os modelos GFS e GEFS (1ํmembro de 21 disponíveis em cada previsão). Estes dois últimos modelos apresentam um desempenho idêntico, embora o modelo GEFS apresente um menor valor da média do erro relativo para 3 dias de previsão.

Os resultados obtidos permitem afirmar que as previsões dos modelos poderão ser utilizadas no suporte à decisão, permitindo uma gestão mais efetiva das infraestruturas de drenagem da bacia do rio Ave. 
Figura 7: Evolução da média dos erros relativos para os modelos de previsão WRF, GFS e GEFS para quatro horizontes de previsão.

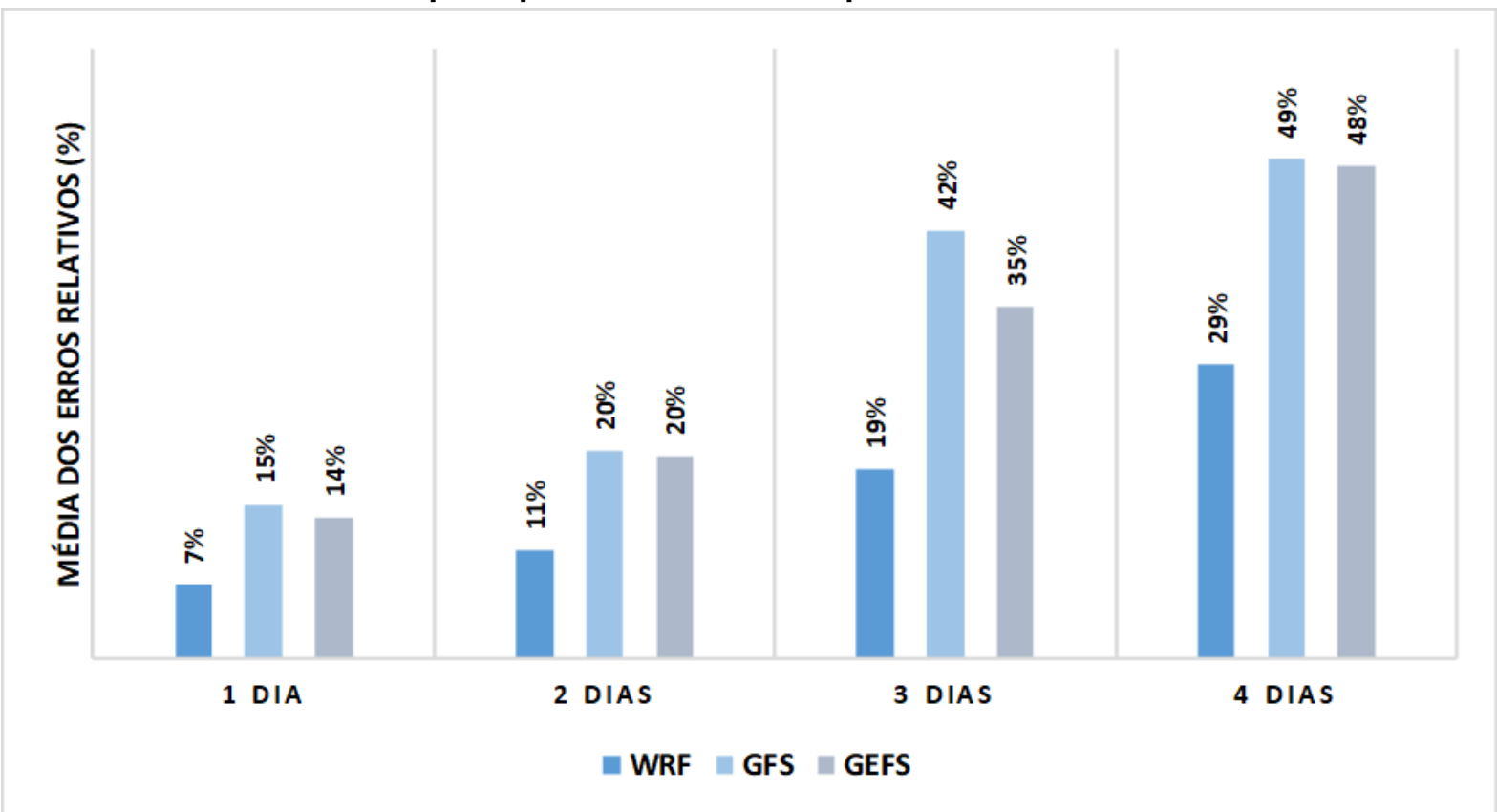

Fonte: Autores, 2020.

\subsection{Operação do sistema de drenagem}

Um dos problemas que afeta a operação do sistema de drenagem de águas residuais domésticas e industriais da bacia do rio Ave é a afluência indevida de caudais de águas pluviais que entram no sistema ou através de infiltrações nas infraestruturas ou através de ligações indevidas de redes de águas pluviais à rede de esgotos.

Tendo por base os dados de precipitação e caudais medidos com resolução horária no ano de 2016, num dos intercetores, é possível estimar padrões de caudais diários para dias secos e dias com ocorrência de precipitação, conforme apresentado na figura 8 .

Como se pode constatar os valores dos caudais transportados neste intercetor têm um incremento significativo em períodos em que ocorre precipitação. Praticamente duplicam o seu valor durante os dias de chuva. Este aumento é bastante problemático, uma vez que todas as ETAR foram concebidas para receber efluentes domésticos $e$ industriais com volumes pouco significativos de águas pluviais infiltradas e como tal, não existindo capacidade de tratamento do volume total afluente, parte deste volume é descarregado para meio recetor sem tratamento. Por outro lado, a aumento das afluências implica que a rede entre em pressão originando derrames não controlados através das tampas das caixas de visita.

Com a disponibilização do sistema de apoio à decisão a operação do sistema tornou-se mais eficiente. De uma operação reativa, tendo por base a comunicação à posteriori de derrames ao longo da rede, passou-se a uma operação preventiva, em que com base na previsão das precipitações se adotam medidas para minorar e controlar as descargas que ocorrem na rede em períodos de chuva intensa. Os sistemas SCADA (caudais) e Delft-FEWS (previsões de precipitação) são utilizados para emissão de alertas.

A título exemplificativo apresentam-se na figura 9 registos de precipitações e caudais no intercetor do Ave para o mês de outubro de 2019. Durante este mês foram emitidos seis alertas (dias $13,14,16,19,29$ e 30). Os alertas foram emitidos com uma antecedência de 48 horas e confirmados com a previsão a 24 horas, tendo por base valores de intensidade de precipitação superiores a 5 $\mathrm{mm} / \mathrm{h}$. Todos os alertas foram verdadeiros conforme se comprova pelas medições de precipitação através do radar.

A partir destes valores de intensidade de precipitação o sistema entra em pressão e o caudal ultrapassa a capacidade máxima de transporte estimada em $500 \mathrm{~m}^{3} / \mathrm{h}$, a partir do modelo hidrodinâmico da rede.

As medidas adotadas após a emissão de alerta, passam por verificar a estanqueidade de tampas de caixas de visita em locais em que 
eventuais descargas apresentam maiores impactos ambientais e criação de condições hidráulicas para permitir descargas em locais mais adequados. Esta solução é naturalmente provisória, e só poderá ser solucionada quando se identificarem as ligações indevidas ou com investimento em infraestruturas adequadas à gestão dos volumes afluentes durante eventos de chuva intensos.

Figura 8: Caudais médios diários no intercetor do Ave para dias secos e dias com precipitação nos diferentes dias da semana.

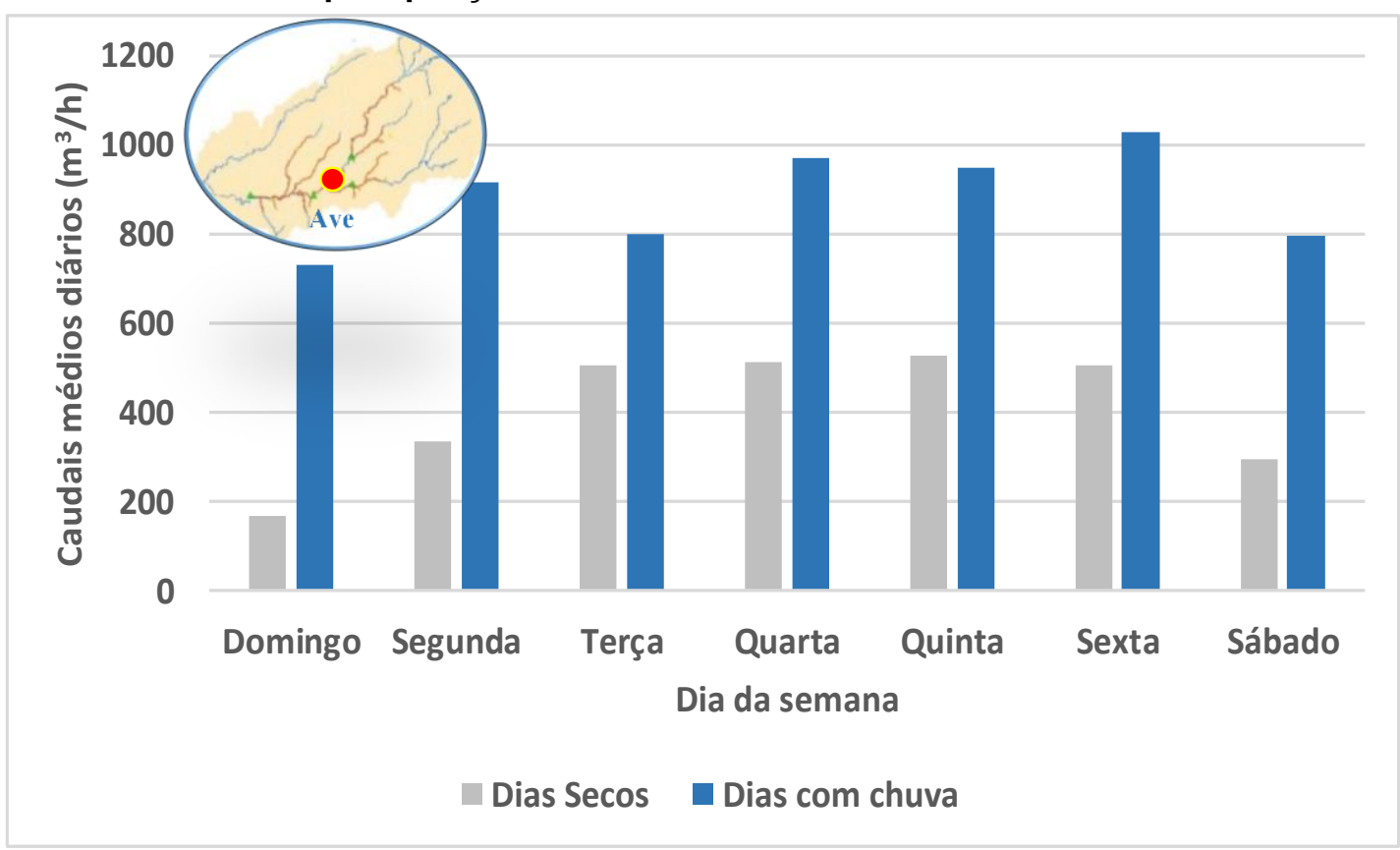

Fonte: Autores, 2020.

Figura 9: Registos utilizados para emissão de alertas (círculos vermelhos) incluindo as previsões WRF de precipitação, de precipitações do radar meteorológico e de caudal durante o mês de outubro de 2019 no intercetor do Ave.

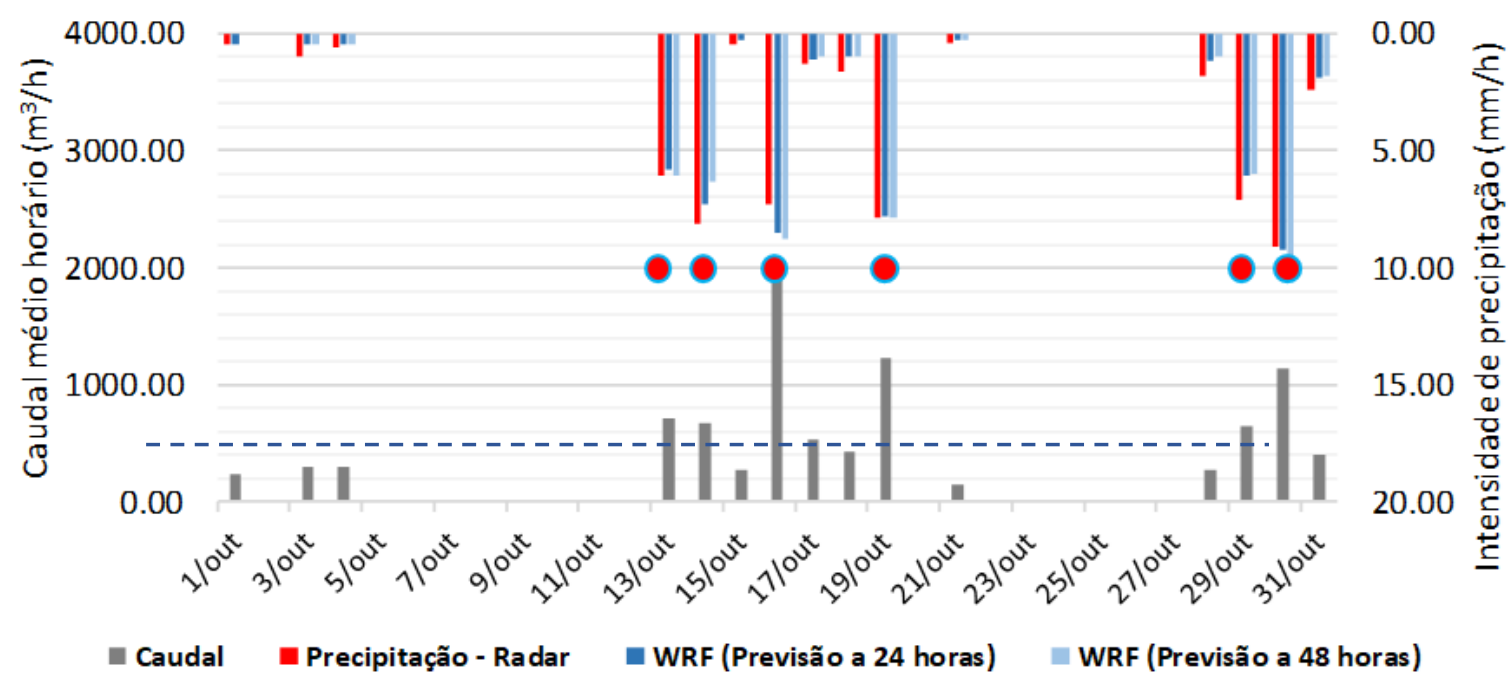

Fonte: Autores, 2020. 


\section{CONCLUSÕES}

O trabalho apresentado, pioneiro a nível nacional e ainda raro em termos internacionais, permitiu aumentar consideravelmente 0 desempenho económico e ambiental da entidade responsável pela operação de um conjunto de infraestruturas de tratamento e drenagem complexos. Pela primeira vez, desenvolveram-se e aplicaram-se ferramentas hidroinformáticas para a gestão operacional de um conjunto de infraestruturas de drenagem na bacia do rio Ave. A integração de diferentes tecnologias e ferramentas hidroinformáticas foi conseguida com sucesso como se pode comprovar pelos resultados apresentados anteriormente.

Foram diagnosticadas as causas responsáveis pelas afluências excessivas durante eventos de precipitação, resultando de forma clara que afluem à rede de recolha de águas residuais domésticas e industriais importantes volumes de águas pluviais provenientes das redes secundárias ligadas à rede principal e que foram quantificados neste trabalho. A análise simultânea de medições de precipitação e hidrogramas medidos na rede principal permite antecipar como a sua origem provável, sistemas de recolha e drenagem de águas pluviais indevidamente ligados à rede de águas residuais domésticas e industriais.

A plataforma Delft-FEWS permitiu aceder, integrar e processar um conjunto de informações relevantes que constitui uma mais valia para os operadores do sistema, salientando-se o acesso a resultados de modelos de previsão meteorológica. Destes, as previsões de precipitação permitem, por exemplo, antecipar a programação de intervenções das equipas de manutenção sempre que as condições meteorológicas assim o exigem.

A avaliação da incerteza associada aos resultados de previsões dos modelos WRF, GFS e GEFS para diferentes horizontes de previsão (de um a quatro dias) permitiu concluir que o modelo WRF apresenta o melhor desempenho. O erro relativo das precipitações diárias é da ordem de $11 \%$ para o modelo WRF para horizontes de dois dias, mas sobe para valores entre $30 \%$ a $50 \%$ para horizontes de quatro dias para os três modelos de previsão analisados. Adotam-se, assim, as previsões com antecedência de dois dias e confirmadas a um dia na operação do sistema de drenagem. Salienta-se o interesse do sistema implementado do ponto de vista operacional, ao permitir antecipar com antecedência eventos extremos com um nível de incerteza razoável.

A ferramenta de apoio à decisão desenvolvida para a bacia do Rio Ave constitui uma plataforma tecnológica robusta e eficiente para apoio à gestão de recursos hídricos. Os resultados obtidos mostram que esta nova ferramenta será extremamente eficaz e importante para atingir os objetivos da gestão operacional à escala da bacia hidrográfica. Nos próximos anos, a utilização deste tipo de ferramentas avançadas de controlo operacional será um requisito essencial para todas as entidades responsáveis pela gestão de recursos hídricos, sendo que cada vez mais, gerir este tipo de recursos se torna mais desafiante e complexo.

\section{AGRADECIMENTOS}

Os autores agradecem à TRATAVE S.A., empresa onde o projeto vem a ser desenvolvido, pela disponibilidade de recursos, sejam eles humanos, tecnológicos ou financeiros.

\section{REFERÊNCIAS}

BUTLER, D., e SCHUTZE, M. (2005). Integrating simulation models with a view to optimal control of urban wastewater systems. Environmental Modelling \& Software, 20(4), 415-426.

DELTARES. (2011). SOBEK - Reference Manual.Delft. Deltares. Delft Hydraulics, Netherlands.

FLETCHER, T., ANDRIEU, H., e HAMEL, P. (2013). Understanding, management and modelling of urban hydrology and its consequences for receiving waters. Advances in Water Resources, 51, 261-279.

FUCHS, L., \& BEENEKEN, T. (2005). Development and implementation of a real-time control strategy for the sewer system of the city of Vienna. Water Science and Technology, 52(5), 187-194.

JOSEPH-DURAN, B., CARLOS, O.-M., \& CEMBRANO, G. (2014). Hybrid modeling and receding horizon control of sewer networks. Water Resources Research, 50, 8497-8514.

KAISER, Ilza Machado (2006). Avaliação de métodos de composição de campos de precipitação para uso em modelos hidrológicos distribuídos. Tese de Doutoramento. Escola de Engenharia de São Carlos da Universidade de São Paulo. Brasil. 399pp.

LOWE, R., VEZZARO, L., STEEN, P., e GRUM., M. (2016). Probabilistic runoff volume forecasting in riskbased optimization for RTC of urban drainage systems. Environmental Modelling Software, 80, 143-158. 
MEDINA, H., TIAN, D., MARIN, F. R., \& CHIRICO, G. B. (2019). Comparing GEFS, ECMWF, and Postprocessing Methods for Ensemble Precipitation Forecasts over Brazil. Journal of Hydrometeorology, 20(4), 773-790.

NICOLAU, R. (2002). Modelação e Mapeamento da Distribuição Espacial da Precipitação-Uma Aplicação a Portugal Continental. Faculdade de Ciências e Tecnologia. Universidade Nova de Lisboa, Lisboa.

PASQUAL, Ana Lucia K., LIPOSKI, Jhonatan e CORSO, Leandro Luis. Aplicação de Cadeias de Markov para Determinação de Probabilidades de Estados Climáticos na Cidade de Caxias do Sul. Caxias do Sul: XXXVI ENCONTRO NACIONAL DE ENGENHARIA DE PRODUÇÃO, 2016.

PEREIRA, A., PINHO, J., FARIA, R., e VIEIRA., J. (2018). A DSS for operational management of. HIC 2018. 13th International Conference on Hydroinformatics (pp. 1616-1623). Palermo, Italy: EPiC Series in Engineering.

PEREIRA, A., PINHO, J., FARIA, R., VIEIRA., J. e COSTA, C. (2019). Improving operational management of wastewater systems. A case study. Water Science and Technology, 8 (1), 173-183.

PINHO, J. L., e VIEIRA., J. M. (2014). A web-based hydroinformatic platform for water quality modelling in a river basin. 11th International Conference on Hydroinformatics (pp. 1-8). New York, USA: CUNY Academic Works.

PINHO, J. L., VIEIRA, J.M., PINHO, R. P., \& ARAÚJO, J. P. (2011). Plataforma Web de suporte ao sistema de modelação da rede hidrográfica da bacia do rio Ave. (Web based technological platform to support river basin modelling system). Revista Recursos Hídricos (Portuguese Water Resources Journal), 32 (1), 13-28.
PUIG, V. C. (2009). Predictive optimal control of sewer networks using CORAL tool: application to Riera Blanca catchment in Barcelona. Water Science and Technology, 60 (4), 869-878.

SHISHEGAR, S., DUCHESNE, S., e PELLETIER, G. (2018). Optimization methods applied to stormwater management problems: a review. Urban Water, 15 (3), 276-286.

SCHWANENBERG, D., TALSMA, J., PINHO, J. L., ASSIS DOS REIS, A., RODRIGUES BESSA, M., \& KUWAJIMA, J. (2013). Short-term reservoir optimization for mitigation downstream flood risks. In 8th International Conference of EWRA "Water Resources Management in an Interdisciplinary and Changing Context", Porto, Portugal.

SCHWANENBERG, D., FAN, F. M., NAUMANN, S., KUWAJIMA, J. I., MONTERO, R. A., \& DOS REIS, A. A. (2015). Short-term reservoir optimization for flood mitigation under meteorological and hydrological forecast uncertainty. Water Resources Management, 29(5), 1635-1651.

SNIRH. Sistema Nacional de Informação de Recursos Hídricos. Disponível em: https://snirh.apambiente.pt (Acedido em 22 outubro 2017).

VAN DAAL-ROMBOUTS, P. D., LANGEVELD, J., e CLEMENS, F. (2016). Integrated real time control of influent pumping station and primary settling tanks at WWTP Eindhoven. In SPN8: Proceedings of the 8th International Conference on Sewer Processes and Networks, (pp. 1-4). Rotterdam, the Netherlands.

WU, W., ZOU, H., SHAN, J., \& WU, S. (2018). A Dynamical ZR Relationship for Precipitation Estimation Based on Radar Echo-Top Height Classification. Advances in Meteorology, 2018. 